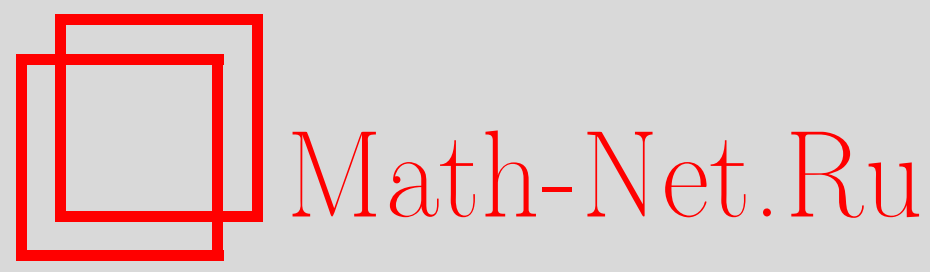

Г. И. Ивченко, В. А. Миронова, Некоторые вопросы спектрального анализа случайных булевых функций с ограничениями, Дискрет. матем., 2013, том 25, выпуск 1, 90-110

DOI: https://doi.org/10.4213/dm1223

Использование Общероссийского математического портала Math-Net.Ru подразумевает, что вы прочитали и согласны с пользовательским соглашением http://www . mathnet.ru/rus/agreement

Параметры загрузки:

IP: 54.166 .219 .16

26 апреля 2023 г., $11: 52: 18$ 


\title{
Некоторые вопросы спектрального анализа случайных булевых функций с ограничениями
}

\author{
() 2013 г. Г. И. Ивченко, В. А. Миронова
}

Исследуются свойства спектра Уолша булевых функций от $n$ переменных, случайно выбираемых из некоторых подмножеств всех таких функций. Выводятся соответствующие характеристические функции спектра и находятся точные и асимптотические (при $n \rightarrow \infty$ ) распределения различных его характеристик.

\section{1. Введение}

Пусть

$$
V_{n}=\left\{v_{0}, v_{1}, \ldots, v_{2^{n-1}}\right\}
$$

есть $n$-мерное векторное пространство над полем из двух элементов, векторы которого упорядочены по возрастанию соответствующих им чисел, где $v_{i}=\left(v_{1 i}, v_{2 i}, \ldots, v_{n i}\right)$ отвечает двоичному представлению числа $i=0,1, \ldots, 2^{n}-1$ :

$$
i=v_{1 i} 2^{n-1}+v_{2 i} 2^{n-2}+\ldots+v_{n-1, i} 2+v_{n i} .
$$

Пусть, далее, $f: V_{n} \rightarrow\{0,1\}$ есть булева функция от $n$ переменных и $F_{n}=\{f\}-$ множество всех таких функций. Любую булеву функцию, как хорошо известно, можно представить в виде вектора

$$
f_{n}=\left(f\left(v_{0}\right), f\left(v_{1}\right), \ldots, f\left(v_{2^{n}-1}\right)\right) \equiv\left(f_{n 0}, f_{n 1}, \ldots, f_{n, 2^{n}-1}\right),
$$

называемого ее таблицей истинности [1].

Целочисленная функция $w_{f}: V_{n} \rightarrow \mathbf{R}$, определяемая соотношением

$$
w_{f}(u)=\sum_{x \in V_{n}} f(x)(-1)^{(u, x)}, \quad u \in V_{n},
$$

называется преобразованием Уолша булевой функции $f$; величина $w_{n i}=w_{f}\left(v_{i}\right)$ называется спектральным коэффициентом функции $f$, отвечающим вектору $v_{i}$, а совокупность всех этих величин - вектор

$$
w_{n}=\left(w_{n 0}, w_{n 1}, \ldots, w_{n, 2^{n}-1}\right)
$$

называется спектром Уолша, или просто спектром функции $f$. 
Напомним также, что символ $\|f\|$ обозначает вес функции $f$ (число единичных компонент вектора (1)), и скалярное произведение векторов $a=\left(a_{1}, \ldots, a_{n}\right)$ и $b=\left(b_{1}, \ldots, b_{n}\right)$ из $V_{n}$ вычисляется по формуле

$$
(a, b)=a_{1} b_{1} \oplus \ldots \oplus a_{n} b_{n},
$$

где $\oplus$ - знак сложения по модулю 2.

Известно, что функция $f$ однозначно определяется своим спектром по формуле обращения:

$$
f(x)=2^{-n} \sum_{u \in V_{n}} w_{f}(u)(-1)^{(u, x)}, \quad x \in V_{n} .
$$

Далее, введем матрицы

$$
\begin{aligned}
& H_{0}=(1), \\
& H_{1}=\left(\begin{array}{rr}
1 & 1 \\
1 & -1
\end{array}\right),
\end{aligned}
$$

и вообще,

$$
H_{k}=\left(\begin{array}{rr}
H_{k-1} & H_{k-1} \\
H_{k-1} & -H_{k-1}
\end{array}\right)=H_{1} \otimes H_{k-1}, \quad k \geqslant 2,
$$

где $\otimes$ означает кронекеровское произведение.

Матрицы такого вида называются матрицами Сильвестра-Адамара [1, с. 396]. Отметим некоторые их свойства, необходимые нам в дальнейшем. Они симметричны:

$$
H_{n}^{\prime}=H_{n}
$$

удовлетворяют условию ортогональности

$$
H_{n} H_{n}=2^{n} I_{2^{n}},
$$

где $I_{r}$ есть единичная матрица порядка $r$, откуда следует, что

$$
H_{n}^{-1}=2^{-n} H_{n}
$$

наконец, имеют место представления

$$
H_{n}=\left((-1)^{\left(v_{i}, v_{j}\right)}\right)=\left(l_{0}^{\prime}, l_{1}^{\prime}, \ldots, l_{2^{n}-1}^{\prime}\right),
$$

где вектор-строка

$$
l_{j}=\left((-1)^{\left(v_{j}, v_{0}\right)},(-1)^{\left(v_{j}, v_{1}\right)}, \ldots,(-1)^{\left(v_{j}, v_{2} n_{-1}\right)}\right)
$$

есть $(+1,-1)$ последовательность для линейной функции

$$
l_{j}(x)=\left(v_{j}, x\right), \quad x \in V_{n} ;
$$

при этом

$$
\sum_{j=0}^{2^{n}-1}(-1)^{\left(v_{j}, v_{i}\right)}= \begin{cases}2^{n}, & i=0 \\ 0, & i>0\end{cases}
$$


в строках и столбцах матрицы $H_{n}$, за исключением первых строки и столбца, одинаковое число 1 и -1 .

В терминах матриц Сильвестра-Адамара, преобразование Уолша (2) и формула обращения (4) могут быть записаны в виде

$$
w_{n}=f_{n} H_{n}, \quad f_{n}=2^{-n} w_{n} H_{n} .
$$

Булевы функции широко используются в задачах защиты информации, и потому они являются популярным объектом математического и криптографического анализа. Соответствующая литература огромна и она достаточно полно отражена в обзорах [2, 3]. Объект нашего интереса - спектр (3) булевой функции: многие криптографические свойства булевой функции выражаются именно в терминах ее спектральных характеристик. В последние годы для исследования спектра (3) весьма эффективно применяется вероятностный подход (см. [4-6] и библиографию в них), когда на множестве $F_{n}=\{f\}$ вводится та или иная вероятностная мера, приписывающая каждой функции этого множества соответствующий вес. Тогда спектр случайно выбранной функции становится случайным вектором, и для исследования различных его особенностей “в среднем” успешно применяются методы теории вероятностей, и в особенности ее предельные теоремы, позволяющие устанавливать полезные асимптотические (при $n \rightarrow \infty$ ) оценки для различных характеристик спектра.

Такой подход позволяет анализировать и ситуации, когда класс используемых булевых функций представляет собой то или иное подмножество множества $F_{n}$, например, подмножество

$$
F_{n k}=\left\{f \in F_{n}:\|f\|=k\right\}
$$

функций заданного веса $k, 0<k<2^{n}$.

Для исследования спектральных свойств функций с таким ограничением естественно вводить вероятностную меру, сосредоточенную лишь на классе $F_{n k}$, например, равномерную, приписывающую каждой функции этого класса вес

$$
\left|F_{n k}\right|^{-1}=\left(\begin{array}{c}
2^{n} \\
k
\end{array}\right)^{-1},
$$

- такую модель мы будем называть кратко моделью $F_{n k}$.

Другой, важный для приложений пример, представляют симметрические булевы функции, иными словами, такие функции, которые принимают одно и то же значение на всех булевых векторах одинакового веса. Обзор общих свойств таких функций, накопленных результатов по их анализу, а также их криптографических применений имеется в $[3,8,9]$. Представляет интерес рассмотреть аналогичные вопросы и для подмножеств симметрических функций с ограничениями, подобными описанным выше.

Настоящая работа и посвящена анализу двух таких моделей с ограничениями. Для каждой модели выводится характеристическая функция спектра Уолша случайной булевой функции, и находятся точные и асимптотические (при $n \rightarrow \infty$ ) распределения различных его характеристик. 


\section{2. Модель $\boldsymbol{F}_{n k}$}

\section{1. Характеристическая функция спектра случайной булевой функции}

Общая конструкция вероятностной модели для булевых функций, предложенная в [5], имеет следующий вид.

Зададим на множестве $F_{n}=\{f\}$ вероятностную меру, приписывающую каждой функции этого множества вес $\mathbf{P}(f)$, и пусть

$$
\varphi_{n}(t)=\mathbf{E} e^{i t f_{n}^{\prime}}, \quad t=t\left(t_{0}, t_{1}, \ldots, t_{2^{n}-1}\right),
$$

есть соответствующая характеристическая функция (вектор $f_{n}$ определен в (1)):

$$
\varphi_{n}(t)=\sum_{f \in F_{n}} e^{i t f_{n}^{\prime}} \mathbf{P}(f)
$$

Тогда характеристическая функция случайного спектра $w_{n}$ (см. (3)) есть

$$
\begin{aligned}
\Phi_{n}(t) & =\mathbf{E} e^{i t w_{n}^{\prime}}=\mathbf{E} \exp \left\{i\left(t H_{n}\right) f_{n}^{\prime}\right\} \\
& =\varphi_{n}\left(t H_{n}\right)=\varphi_{n}\left(t l_{0}^{\prime}, t l_{1}^{\prime}, \ldots, t l_{2^{n-1}}^{\prime}\right) .
\end{aligned}
$$

Соотношениями (9) и (10) и задается общая конструкция вероятностной модели на множестве всех булевых функций $F_{n}=\{f\}$, позволяющая, в принципе, проводить анализ спектра $w_{n}$ для различных конкретных вариантов задания меры $\mathbf{P}(f)$. В [5] рассмотрен и детально проанализирован вариант, когда компоненты вектора $f_{n}$ являются независимыми и одинаково распределенными бернуллиевскими случайными величинами с произвольным параметром. Здесь мы рассматриваем определенную во введении модель $F_{n k}$, когда мера $\mathbf{P}(f)$ сосредоточена на подмножестве булевых функций $F_{n k}=\left\{f \in F_{n}:\|f\|=k\right\}$ и она равномерная:

$$
\mathbf{P}(f)=\left|F_{n k}\right|^{-1}=\left(\begin{array}{c}
2^{n} \\
k
\end{array}\right)^{-1}, \quad f \in F_{n k} .
$$

Для этого случая характеристическая функция $\varphi_{n}(t)=\varphi_{n k}(t)$ принимает вид

$$
\varphi_{n k}(t)=\sum_{f \in F_{n k}} e^{i t f_{n}^{\prime}} \mathbf{P}(f)=\left(\begin{array}{c}
2^{n} \\
k
\end{array}\right)^{-1} \sum_{0 \leqslant j_{1}<j_{2}<\ldots<j_{k} \leqslant 2^{n}-1} e^{i\left(t_{j_{1}}+t_{j_{2}}+\ldots+t_{j_{k}}\right)} .
$$

Здесь многомерная сумма может быть записана как коэффициент при $z^{k}$ произведения $\prod_{j=0}^{2^{n}-1}\left(1+z e^{i t_{j}}\right)$, поэтому, используя оператор $\left[z^{k}\right]=\operatorname{coef}_{z}$, получаем, что

$$
\varphi_{n k}(t)=\left(\begin{array}{c}
2^{n} \\
k
\end{array}\right)^{-1}\left[z^{k}\right] \prod_{j=0}^{2^{n}-1}\left(1+z e^{i t_{j}}\right)
$$

Наконец, для характеристической функции $\Phi_{n}(t)=\Phi_{n k}(t)$ спектра такой случайной булевой функции, выбираемой с равной вероятностью из множества $F_{n k}$, из (10) имеет место представление

$$
\Phi_{n k}(t)=\left(\begin{array}{c}
2^{n} \\
k
\end{array}\right)^{-1}\left[z^{k}\right] \prod_{j=0}^{2^{n}-1}\left(1+z e^{i t l_{j}^{\prime}}\right)
$$


где векторы $l_{j}$ определены в (6).

Это представление является основой для анализа распределений различных характеристик спектра, чему посвящены следующие разделы.

\section{2. Моменты спектра}

Мы начинаем анализ модели $F_{n k}$ с нахождения моментов спектра $w_{n}$ (см. (3)). Это может быть сделано прямыми вычислениями.

Прежде всего, найдем первые и вторые моменты вектора $f_{n}$, определенного в (1).

Из (11) получаем, что для любого $j$

$$
\mathbf{E} f_{n j}=\mathbf{P}\left(f_{n j}=1\right)=\left(\begin{array}{c}
2^{n}-1 \\
k-1
\end{array}\right) /\left(\begin{array}{c}
2^{n} \\
k
\end{array}\right)=\frac{k}{2^{n}}
$$

и при $j \neq s$

$$
\mathbf{E} f_{n j} f_{n s}=\mathbf{P}\left(f_{n j}=f_{n s}=1\right)=\left(\begin{array}{c}
2^{n}-2 \\
k-2
\end{array}\right) /\left(\begin{array}{c}
2^{n} \\
k
\end{array}\right)=\frac{k(k-1)}{2^{n}\left(2^{n}-1\right)}
$$

Отсюда

$$
\begin{gathered}
\mathbf{D} f_{n j}=\mathbf{E} f_{n j}-\left(\mathbf{E} f_{n j}\right)^{2}=\frac{k\left(2^{n}-k\right)}{2^{2 n}}, \\
\operatorname{cov}\left(f_{n j}, f_{n s}\right)=\mathbf{E} f_{n j} f_{n s}-\mathbf{E} f_{n j} \mathbf{E} f_{n s}=-\frac{k\left(2^{n}-k\right)}{2^{2 n}\left(2^{n}-1\right)}, \quad j \neq s .
\end{gathered}
$$

Обозначим

$$
c_{n k}=\frac{k\left(2^{n}-k\right)}{2^{2 n}}, \quad a_{n}=\frac{1}{2^{n}-1},
$$

и введем матрицу

$$
B_{n}=\left(1+a_{n}\right) I_{2^{n}}-a_{n} E_{2^{n}},
$$

где, напомним, $I_{r}$ - единичная матрица порядка $r$, а $E_{r}$ - матрица порядка $r$, все элементы которой равны 1.

Суммируя результаты (15)-(16), можно сформулировать следующее утверждение.

Теорема 1. В модели $F_{n k}$ первые и вторые моменты случайной булевой функции имеют вид

$$
\mathbf{E} f_{n}=\frac{k}{2^{n}} l_{0}, \quad \mathbf{D} f_{n}=c_{n k} B_{n},
$$

где вектор $l_{0}=(1, \ldots, 1)$, см. (5), а матрица $B_{n}$ определена в (18).

Аналогичное утверждение для спектра $w_{n}$ имеет следующий вид. 
Теорема 2. В модели $F_{n k}$ первые и вторые моменты спектра случайной булевой функции равнь:

$$
\begin{aligned}
& \mathbf{E} w_{n}=(k, 0, \ldots, 0), \\
& \mathbf{D} w_{n}=\frac{k\left(2^{n}-k\right)}{2^{n}-1} I_{2^{n}}^{(0)},
\end{aligned}
$$

где $I_{r}^{(0)}$ означает единичную матрицу, у которой первый диагональный элемент заменен на 0.

Доказательство. Поскольку спектр $w_{n}$ есть линейное преобразование (8) вектора $f_{n}$, то

$$
\mathbf{E} w_{n}=\left(\mathbf{E} f_{n}\right) H_{n}, \quad \mathbf{D} w_{n}=H_{n}\left(\mathbf{D} f_{n}\right) H_{n}
$$

(здесь учтено свойство симметричности матрицы $H_{n}$ ).

Формула (20) сразу следует из (19) и свойства ортогональности матрицы $H_{n}$.

Вычислим теперь матрицу (см. (18))

$$
H_{n} B_{n} H_{n}=\left(1+a_{n}\right) H_{n} H_{n}-a_{n} H_{n} E_{2^{n}} H_{n} .
$$

Здесь, напомним,

$$
H_{n} H_{n}=2^{n} I_{2^{n}}
$$

и непосредственно проверяется, что произведение $H_{n} E_{2^{n}} H_{n}$ есть матрица $2^{2 n} C_{n}$ где, в свою очередь, $C_{n}$ есть матрица, у которой лишь первый диагональный элемент равен 1 , а все остальные элементы равны 0. С учетом этого, формула (23) принимает вид

$$
\begin{aligned}
H_{n} B_{n} H_{n} & =\left(1+a_{n}\right) 2^{n} I_{2^{n}}-2^{2 n} a_{n} C_{n} \\
& =\frac{2^{2 n}}{2^{n}-1}\left(I_{2^{n}}-C_{n}\right) .
\end{aligned}
$$

Отсюда, с учетом соотношений (22), (19) и (17), следует формула для дисперсии (21). Теорема доказана.

Добавим некоторые комментарии к полученному результату.

То, что первая компонента вектора $w_{n}$ имеет вырожденное в точке $k$ распределение, конечно же, сразу следует из определения (2):

$$
w_{n 0}=w_{f}(0)=\sum_{x \in V_{n}} f(x)=\|f\|=k, \quad f \in F_{n k} .
$$

Что касается остальных компонент спектра, то они в рассматриваемой модели оказываются центрированными в нуле, некоррелированными и имеющими одинаковые дисперсии. При этом дисперсия

$$
\mathbf{D} w_{n j}=\frac{k\left(2^{n}-k\right)}{2^{n}-1}, \quad j \geqslant 1,
$$

как функция параметра $k$, достигает своего максимального значения $2^{2(n-1)} /\left(2^{n}-1\right)$ при $k=2^{n-1}$; функции с таким весом называются сбалансированными. 


\section{3. Распределения спектральных коэффициентов}

Перейдем теперь к вопросу о распределениях спектральных характеристик в модели $F_{n k}$ и прежде всего найдем распределение отдельных компонент вектора $w_{n}$. Мы уже знаем, что первая компонента $w_{n 0}$ имеет вырожденное в точке $k$ распределение. Для характеристической функции любого другого спектрального коэффициента $w_{n s}, s \geqslant 1$, из общей характеристической функции (14) приходим к представлению

$$
\mathbf{E} e^{i t_{s} w_{n s}}=\left.\Phi_{n k}(t)\right|_{\substack{t_{j}=0 \\
j \neq s}}=\left(\begin{array}{c}
2^{n} \\
k
\end{array}\right)^{-1}\left[z^{k}\right] \prod_{j=0}^{2^{n}-1}\left(1+z e^{i t_{s} l_{j}(s)}\right),
$$

где $l_{j}(s)-s$-я компонента вектора $l_{j}$.

Но, в силу (7), при $s \geqslant 1$ среди чисел $l_{j}(s), j=0,1, \ldots, 2^{n}-1$, половина 1 и половина -1 , поэтому

$$
\begin{aligned}
\mathbf{E} e^{i t_{s} w_{n s}} & =\left(\begin{array}{c}
2^{n} \\
k
\end{array}\right)^{-1}\left[z^{k}\right]\left(1+z e^{i t_{s}}\right)^{2^{n-1}}\left(1+z e^{-i t_{s}}\right)^{2^{n-1}} \\
& =\left(\begin{array}{c}
2^{n} \\
k
\end{array}\right)^{-1} \sum_{r=0}^{k}\left(\begin{array}{c}
2^{n-1} \\
r
\end{array}\right)\left(\begin{array}{c}
2^{n-1} \\
k-r
\end{array}\right) e^{i(2 r-k) t_{s}} \\
& =\sum_{r=0}^{k} h\left(r ; 2^{n-1}, 2^{n-1}, k\right) e^{i(2 r-k) t_{s}}
\end{aligned}
$$

где $H\left(2^{n-1}, 2^{n-1}, k\right)=\left\{h\left(r ; 2^{n-1}, 2^{n-1}, k\right), r=0,1, \ldots, k\right\}-$ гипергеометрическое распределение с параметрами $\left(2^{n-1}, 2^{n-1}, k\right)$.

Представление (24) дает вид одномерных распределений спектральных коэффициентов, что мы сформулируем в виде следующей теоремы.

Теорема 3. В модели $F_{n k}$ случайная величина $\tilde{w}_{n s}=\frac{1}{2}\left(w_{n s}+k\right)$ nри всех $s=1, \ldots, 2^{n}-1$ имеет гипергеометрическое распределение $H\left(2^{n-1}, 2^{n-1}, k\right)$.

Таким образом, в рассматриваемой модели все спектральные коэффициенты, за исключением первого, имеют сдвинутое на величину $k$ симметричное относительно нуля гипергеометрическое распределение на решетке целых чисел одинаковой четности с числом $k$ :

$$
\mathbf{P}\left(w_{n s}+k=2 r\right)=h\left(r ; 2^{n-1}, 2^{n-1}, k\right), \quad r=0,1, \ldots, k .
$$

\subsection{1. О моментах}

В дополнение к теореме 2 отметим, что все нечетные моменты случайных величин $w_{n s}$, $s \geqslant 1$, равны нулю, а их четные моменты можно находить, используя известные соотношения для факториальных моментов гипергеометрического распределения:

$$
\mathbf{E}\left(\widetilde{w}_{n s}\right)_{j}=(k)_{j}\left(2^{n-1}\right)_{j} /\left(2^{n}\right)_{j}, \quad j \geqslant 1,
$$

где

$$
(a)_{j}=a(a-1)(a-2) \cdots(a-j+1) .
$$




\subsection{2. Сходимость к биномиальному распределению}

Используя известные асимптотики для гипергеометрического распределения, можно легко установить соответствующие предельные теоремы для спектральных коэффициентов.

Теорема 4. Пусть $n \rightarrow \infty$, а параметр $k$ фиксирован. Тогда

$$
\begin{array}{crl}
\mathbf{D} w_{n s}=k+O\left(2^{-n}\right), & s \geqslant 1, \\
\mathbf{P}\left(w_{n s}+k=2 r\right)=\left(\begin{array}{l}
k \\
r
\end{array}\right)\left(\frac{1}{2}\right)^{k}\left(1+\frac{b}{2^{n}}+O\left(2^{-2 n}\right)\right), & 0 \leqslant r \leqslant k,
\end{array}
$$

где

$$
b=\frac{r}{2}-2\left(r-\frac{k}{2}\right)^{2}
$$

В силу симметрии распределения случайных величин $w_{n s}, s \geqslant 1$, сходимость к биномиальному распределению будет иметь место и в случае очень больших $k$, именно, когда $2^{n}-k$ конечно.

\subsection{3. Сходимость к нормальному распределению}

Имеет место следующая локальная предельная теорема.

Теорема 5. Пусть $n, k, 2^{n}-k \rightarrow \infty$, тогда

$$
\mathbf{P}\left(\frac{w_{n s}}{\sqrt{k\left(1-k 2^{-n}\right)}}=m\right) \rightarrow \frac{1}{\sqrt{2 \pi}} e^{-m^{2} / 2}, \quad|m| \leqslant c<\infty .
$$

На этом же пути можно получить и двумерные распределения, хотя это потребует уже больших усилий.

Рассмотрим две произвольные компоненты спектра $\left(w_{n r}, w_{n s}\right), 1 \leqslant r<s \leqslant 2^{n}-1$, тогда их совместная характеристическая функция получается из (14) при подстановке $t_{j}=0, j \neq r, s$, и имеет вид

$$
\begin{aligned}
\psi_{n k}\left(t_{r}, t_{s}\right) & =\mathbf{E} e^{i\left(t_{r} w_{n r}+t_{s} w_{n s}\right)} \\
& =\left(\begin{array}{c}
2^{n} \\
k
\end{array}\right)^{-1}\left[z^{k}\right] \prod_{j=0}^{2^{n}-1}\left(1+z e^{i\left(t_{r} l_{j}(r)+t_{s} l_{j}(s)\right)}\right) .
\end{aligned}
$$

В силу свойства ортогональности матриц Сильвестра-Адамара, среди пар $\left(l_{j}(r), l_{j}(s)\right)$, $j=0,1, \ldots, 2^{n}-1$, каждая комбинация $(1,1),(1,-1),(-1,1)$ и $(-1,-1)$ встречается одинаковое число раз, равное $2^{n-2}$, поэтому произведение в (29) принимает вид $\prod_{\beta_{1}, \beta_{2}= \pm 1}\left(1+z e^{i\left(\beta_{1} t_{r}+\beta_{2} t_{s}\right)}\right)^{2^{n-2}}$. Раскладывая здесь каждый из четырех сомножителей по степеням $z$ и выделяя, в соответствии с (29), коэффициент при $z^{k}$, получим представление

$$
\psi_{n k}\left(t_{r}, t_{s}\right)=\left(\begin{array}{c}
2^{n} \\
k
\end{array}\right)^{-1} \sum_{m_{1}+\ldots+m_{4}=k} \prod_{j=1}^{4}\left(\begin{array}{c}
2^{n-2} \\
m_{j}
\end{array}\right) e^{i t_{r}\left(m_{1}+m_{2}-m_{3}-m_{4}\right)+i t_{s}\left(m_{1}-m_{2}+m_{3}-m_{4}\right)},
$$


которое, в свою очередь, заменой $m_{1}+m_{2}-m_{3}-m_{4}=u, m_{1}-m_{2}+m_{3}-m_{4}=v$ приводится к виду

$$
\begin{aligned}
\psi_{n k}\left(t_{r}, t_{s}\right)= & \left(\begin{array}{c}
2^{n} \\
k
\end{array}\right) \sum_{m, u, v}\left(\begin{array}{c}
2^{n}-2 \\
m
\end{array}\right) \\
& \times\left(\begin{array}{c}
2^{n-2} \\
m-(u+v) / 2
\end{array}\right)\left(\begin{array}{c}
2^{n}-2 \\
(k+u) / 2-m
\end{array}\right)\left(\begin{array}{c}
2^{n}-2 \\
(k+v) / 2-m
\end{array}\right) e^{i t_{r} u+i t_{s} v} .
\end{aligned}
$$

Это и есть окончательное выражение для совместной характеристической функции пары спектральных коэффициентов, дающее одновременно и явный вид распределения этой пары, поскольку

$$
\psi_{n k}\left(t_{r}, t_{s}\right)=\sum_{u, v} \mathbf{P}\left(w_{n r}=u, w_{n r}=v\right) e^{i\left(t_{r} u+t_{s} v\right)}
$$

Соответствующее утверждение о виде этого распределения формулируется следующим образом.

Теорема 6. В модели $F_{n k}$ для любых $(r, s), 1 \leqslant r<s \leqslant 2^{n}-1$, справедливо соотночение

$$
\begin{aligned}
\mathbf{P}\left(w_{n r}=u, w_{n s}=v\right)= & \left(\begin{array}{c}
2^{n} \\
k
\end{array}\right) \sum_{m}\left(\begin{array}{c}
2^{n}-2 \\
m
\end{array}\right) \\
& \times\left(\begin{array}{c}
2^{n-2} \\
m-(u+v) / 2
\end{array}\right)\left(\begin{array}{c}
2^{n}-2 \\
(k+u) / 2-m
\end{array}\right)\left(\begin{array}{c}
2^{n}-2 \\
(k+v) / 2-m
\end{array}\right) .
\end{aligned}
$$

Отметим некоторые свойства этого распределения.

(1) Вероятности (31) отличны от нуля лишь для значений $u$ и $v$, одинаковой четности с числом $k$.

(2) Вероятности (31) не меняются при изменении знаков у переменных $u$ и $v$ (свойство симметрии).

(3) Используя формулы для гипергеометрического распределения (см. (24)), распределение (31) можно записать также в виде

$$
\begin{aligned}
\mathbf{P}\left(w_{n r}+k=2 i, w_{n s}\right. & +k=2 j)=h\left(j ; 2^{n-1}, 2^{n-1}, k\right) \\
& \times \sum_{m=0}^{c} h\left(m ; 2^{n-2}, 2^{n-2}, j\right) h\left(i-m ; 2^{n-2}, 2^{n-2}, k-j\right),
\end{aligned}
$$

где $c=\min (i, j, i+j-k)$.

\section{4. Линейные функционалы от спектра}

Простейшими функционалами от спектра $w_{n}$ являются линейные комбинации спектральных коэффициентов

$$
L_{n k}(c)=c w_{n}^{\prime}=\sum_{j=0}^{2^{n}-1} c_{j} w_{n j}, \quad c=\left(c_{0}, c_{1}, \ldots, c_{2^{n}-1}\right) .
$$


Характеристическая функция произвольного линейного функционала допускает представление (см. (14))

$$
\mathbf{E} e^{i \tau L_{n k}(c)}=\mathbf{E} e^{i \tau c w_{n}^{\prime}}=\Phi_{n k}(\tau c)=\left(\begin{array}{c}
2^{n} \\
k
\end{array}\right)^{-1}\left[z^{k}\right] \prod_{j=0}^{2^{n}-1}\left(1+z e^{i \tau c l_{j}^{\prime}}\right) .
$$

Эта, вообще говоря, весьма сложная формула значительно упрощается при выборе коэффициентов $c$ в виде $c=l_{r}, r=0,1, \ldots, 2^{n}-1$, поскольку, в силу ортогональности матриц Сильвестра-Адамара,

$$
l_{r} l_{j}^{\prime}= \begin{cases}2^{n}, & r=j \\ 0, & r \neq j\end{cases}
$$

Таким образом, из (34) следует, что

$$
\begin{aligned}
\mathbf{E} e^{i \tau L_{n k}\left(l_{\tau}\right)} & =\left(\begin{array}{c}
2^{n} \\
k
\end{array}\right)^{-1}\left[z^{k}\right]\left(1+z e^{i \tau 2^{n}}\right)(1+z)^{2^{n}-1} \\
& =\left(\begin{array}{c}
2^{n} \\
k
\end{array}\right)^{-1}\left(\left(\begin{array}{c}
2^{n}-1 \\
k
\end{array}\right)+\left(\begin{array}{c}
2^{n}-1 \\
k-1
\end{array}\right) e^{i \tau 2^{n}}\right) \\
& =\left(1-\frac{k}{2^{n}}\right)+\frac{k}{2^{n}} e^{i \tau 2^{n}} .
\end{aligned}
$$

В итоге мы приходим к следующему утверждению.

Теорема 7. В модели $F_{n k}$ для любого $r, 0 \leqslant r \leqslant 2^{n}-1$, линейный функционал

$$
L_{n k}\left(1_{r}\right)=1_{r} w_{n}^{\prime}
$$

имеет двухточечное распределение вида

$$
\begin{aligned}
\mathbf{P}\left(L_{n k}\left(l_{r}\right)=0\right) & =1-\frac{k}{2^{n}}, \\
\mathbf{P}\left(L_{n k}\left(l_{r}\right)=2^{n}\right) & =\frac{k}{2^{n}}
\end{aligned}
$$

при этом

$$
\begin{aligned}
& \mathbf{E} L_{n k}\left(l_{r}\right)=k, \\
& \mathbf{D} L_{n k}\left(l_{r}\right)=k\left(2^{n}-k\right) .
\end{aligned}
$$

Добавим к этому результату некоторые комментарии. Двухточечность распределений рассматриваемых функционалов непосредственно следует из представления (8), так как всегда

$$
L_{n}^{\prime} \equiv\left(L_{n k}\left(l_{0}\right), \ldots, L_{n k}\left(l_{2^{n}-1}\right)\right)^{\prime}=H_{n} w_{n}^{\prime}=H_{n} H_{n}^{\prime} f_{n}^{\prime}=2^{n} f_{n}^{\prime}
$$

другими словами, сумма $l_{r} w_{n}^{\prime}$ всегда принимает лишь значения 0 и $2^{n}$, а соответствующие вероятности уже зависят от выбора модели. В целом же, распределение вектора $2^{-n} L_{n}$, определенного в (37), всегда совпадает с распределением вектора (таблицы истинности) $f_{n}$. 
Выделим еще частный случай для суммы всех спектральных коэффициентов

$$
L_{n k}\left(l_{0}\right)=\sum_{j=0}^{2^{n}-1} w_{n j}
$$

Для рассматриваемой модели $F_{n k}$ в этой сумме первое слагаемое $w_{n 0}$ не случайно и равно $k$; таким образом, сумма остальных слагаемых имеет двухточечное распределение

$$
\mathbf{P}\left(\sum_{j=1}^{2^{n}-1} w_{n j}=2^{n}-k\right)=1-\mathbf{P}\left(\sum_{j=1}^{2^{n}-1} w_{n j}=-k\right)=\frac{k}{2^{n}} .
$$

Наконец, для случая сбалансированных функций (при $k=2^{n-1}$ ) теорема 7 принимает следующий вид: случайные величины $2^{-n} L_{n, 2^{n-1}}\left(l_{r}\right)$ имеют одно и то же распределение Бернулли $B i(1,1 / 2)$.

Еще один тип линейных функционалов, распределения которых могут быть точно описаны, представляет собой частичные суммы

$$
S_{n k}(r)=\sum_{j=0}^{2^{r}-1} w_{n j}, \quad r=1,2, \ldots, n .
$$

Теорема 8. В модели $F_{n k}$ случайные величин $2^{-r} S_{n k}(r)$ имеют гипергеометрическое распределение $H\left(2^{n-r}, 2^{n}-2^{n-r}, k\right)$ :

$$
\mathbf{P}\left(2^{-r} S_{n k}(r)=j\right)=h\left(j ; 2^{n-r}, 2^{n}-2^{n-r}, k\right), \quad j=0,1, \ldots, k .
$$

Доказательство. В силу рекуррентного свойства (5) матриц Сильвестра-Адамара, в матрице $H_{n}$ ее первые $2^{r}$ строк представляют собой последовательные повторения блока $H_{r}$, и число таких повторений равно $2^{n-r}$. Поэтому, обозначив через $l_{j}(r), j=0,1, \ldots 2^{r}-1$, строки матрицы $H_{r}$, из (34) получаем, что

$$
\begin{aligned}
\mathbf{E} e^{i \tau S_{n k}(r)}=\mathbf{E} e^{i \tau l_{0}(r) w_{n}^{\prime}} & =\left(\begin{array}{c}
2^{n} \\
k
\end{array}\right)^{-1}\left[z^{k}\right] \prod_{j=0}^{2^{r}-1}\left(1+z e^{i \tau l_{0}(r) l_{j}^{\prime}(r)}\right)^{2^{n}-r} \\
& =\left(\begin{array}{c}
2^{n} \\
k
\end{array}\right)^{-1}\left[z^{k}\right]\left[\left(1+z e^{i \tau 2^{\tau}}\right)(1+z)^{2^{r}-1}\right]^{2^{n-r}} \\
& =\left(\begin{array}{c}
2^{n} \\
k
\end{array}\right)^{-1} \sum_{j=0}^{k}\left(\begin{array}{c}
2^{n-r} \\
j
\end{array}\right)\left(\begin{array}{c}
2^{n}-2^{n-r} \\
k-j
\end{array}\right) e^{i j \tau 2^{r}},
\end{aligned}
$$

что эквивалентно (40).

Отметим, что здесь экстремальные случаи $r=1$ и $r=n$ соответствуют утверждениям предыдущих теорем 3 и 7.

\section{5. Нулевые компоненты спектра}

Важной для приложений является такая характеристика спектра, как число его нулевых компонент:

$$
\mu_{0}(n, k)=\sum_{j=0}^{2^{n}-1} I\left(w_{n j}=0\right)
$$


здесь $I(A)$ означает индикатор события $A$.

Из формулы (25) следует, что в рассматриваемой модели спектральные коэффициенты $w_{n j}$ при $j \geqslant 1$ могут принимать нулевые значения лишь при четных $k$ (напомним, что всегда $w_{n 0}=k$, и здесь предполагается, что $\left.k>0\right)$. Поэтому далее считаем, что $k$ четно. Исследование функционала (41) представляет уже гораздо более сложную задачу, и мы ограничимся здесь лишь отысканием его среднего и дисперсии (более полный анализ его распределения ждет своего решения).

С учетом (25), среднее находится просто:

$$
\mathbf{E} \mu_{0}(n, k)=\left(2^{n}-1\right) \mathbf{P}\left(w_{n 1}=0\right)=\left(2^{n}-1\right) h\left(\frac{k}{2} ; 2^{n-1}, 2^{n-1}, k\right) .
$$

Можно показать (рутинные выкладки опускаем), что как функция параметра $k$ это среднее достигает своего минимального значения при $k=2^{n-1}$, то есть для сбалансированных функций. При этом

$$
\begin{aligned}
\mathbf{E} \mu_{0}\left(n, 2^{n-1}\right) & =\left(2^{n}-1\right) h\left(2^{n-2} ; 2^{n-1}, 2^{n-1}, 2^{n-1}\right) \\
& \sim \frac{1}{\sqrt{\pi}} 2^{(n+3) / 2}
\end{aligned}
$$

(асимптотика справедлива при больших значениях $n$ ). Таким образом, для сбалансированных функций число нулевых компонент спектра имеет в среднем порядок квадратного корня из их общего числа $2^{n}$.

Далее положим для краткости, $I_{j}=I\left(w_{n j}=0\right)$. Тогда из (41) получаем, что

$$
\mathbf{D} \mu_{0}(n, k)=\sum_{j=1}^{2^{n}-1} \mathbf{D} I_{j}+\sum_{r \neq s} \operatorname{cov}\left(I_{r}, I_{s}\right)
$$

Здесь

$$
\begin{aligned}
\mathbf{D} I_{j} & =\mathbf{P}\left(w_{n 1}=0\right)\left(1-\mathbf{P}\left(w_{n 1}=0\right)\right) \\
& =h\left(\frac{k}{2} ; 2^{n-1}, 2^{n-1}, k\right)\left(1-h\left(\frac{k}{2} ; 2^{n-1}, 2^{n-1}, k\right)\right) \equiv a_{n k},
\end{aligned}
$$

и при $r \neq s$ (см. (32))

$$
\begin{aligned}
\operatorname{cov}\left(I_{r}, I_{s}\right)= & \mathbf{P}\left(w_{n r}=0, w_{n s}=0\right)-\mathbf{P}\left(w_{n r}=0\right) \mathbf{P}\left(w_{n s}=0\right) \\
= & h\left(\frac{k}{2} ; 2^{n-1}, 2^{n-1}, k\right) \\
& \times \sum_{m}\left(h\left(m ; 2^{n-2}, 2^{n-2}, \frac{k}{2}\right)\right)^{2}-\left(h\left(\frac{k}{2} ; 2^{n-1}, 2^{n-1}, k\right)\right)^{2} \equiv b_{n k} .
\end{aligned}
$$

С учетом этих обозначений, окончательное выражение для дисперсии принимает вид

$$
\mathbf{D} \mu_{0}(n, k)=\left(2^{n}-1\right) a_{n k}+\left(2^{n}-1\right)\left(2^{n}-2\right) b_{n k} .
$$




\section{3. Модель $S_{n r}$}

\section{1. Определение модели и многочлены Кравчука}

Пусть $S_{n}$ - множество симметрических булевых функций от $n$ переменных $f(x), x=$ $\left(x_{1}, x_{2}, \ldots, x_{n}\right) \in V_{n}=\{0,1\}^{n}$, другими словами, таких функций, которые принимают одно и то же значение на всех векторах $x$ одинакового веса

$$
\|x\|=\sum_{i=1}^{n} x_{i} .
$$

Симметрические функции находят широкое применение в криптографической теории и практике, и их анализу посвящено значительное число работ (см. $[3,8,9]$ и библиографию в них).

Обозначим $f(x)=f_{k}$, если $\|x\|=k, k=0,1, \ldots, n$. Тогда, вместо таблицы истинности (вектора размерности $2^{n}$ ), для симметрической функции $f$ можно использовать более экономное ее представление через двоичный вектор $\bar{f}_{n}=\left(f_{0}, f_{1}, \ldots, f_{n}\right)$ размерности $n+1$. Напомним кратко некоторые элементы спектральной теории таких функций.

Преобразование Уолша (2) произвольной симметрической функции $f$ также является симметрической функцией и, обозначив $w_{f}(u)=w_{m}$, если $\|u\|=m$, спектральные коэффициенты $w_{m}$ в рассматриваемом случае можно записать в виде

$$
w_{m}=\sum_{k=0}^{n} f_{k} P_{k}(m ; n), \quad m=0,1, \ldots, n,
$$

где

$$
P_{k}(m ; n)=\sum_{j}(-1)^{j}\left(\begin{array}{c}
m \\
j
\end{array}\right)\left(\begin{array}{l}
n-m \\
k-j
\end{array}\right)
$$

есть известные в анализе многочлены Кравчука [6].

Будем далее под спектром симметрической функции $f$ понимать вектор

$$
\bar{w}_{n}=\left(w_{0}, w_{1}, \ldots, w_{n}\right) .
$$

Тогда с использованием матрицы Кравчука

$$
P_{n}=\left(P_{k}(m ; n)\right)_{k, m=0,1, \ldots, n},
$$

систему (45) можно записать в матричном виде:

$$
\bar{w}_{n}=\bar{f}_{n} P_{n},
$$

а формулу обращения - в виде

$$
\bar{f}_{n}=2^{-n} \bar{w}_{n} P_{n}
$$

Из этих соотношений видно, что в рассматриваемой модели ключевую роль играют свойства многочленов Кравчука; эти свойства достаточно детально описаны в [6] и будут нами использованы по ходу изложения без дополнительных ссылок.

Далее, пусть на множестве $S_{n}$ задана некоторая вероятностная мера $\mathbf{P}$, приписывающая каждому вектору $\bar{f}_{n}=\left(f_{0}, f_{1}, \ldots, f_{n}\right)$ вес $\mathbf{P}\left(\bar{f}_{n}\right)$ и

$$
\varphi_{n}(\bar{t})=\mathbf{E} e^{i \bar{t} \bar{f}_{n}^{\prime}}, \quad \bar{t}=\left(t_{0}, t_{1}, \ldots, t_{n}\right),
$$


есть соответствующая характеристическая функция. Тогда характеристическая функция спектра $\bar{w}_{n}$ с учетом (48) принимает вид

$$
\begin{aligned}
\Phi_{n}(\bar{t}) & =\mathbf{E} e^{i \bar{t} \bar{w}_{n}^{\prime}}=\mathbf{E} e^{i\left(\bar{t} P_{n}^{\prime}\right) \bar{f}_{n}^{\prime}}=\varphi_{n}\left(\bar{t} P_{n}^{\prime}\right) \\
& =\varphi_{n}\left(\sum_{m=0}^{n} t_{m} P_{k}(m ; n), k=0,1, \ldots, n\right) .
\end{aligned}
$$

Это общее представление может быть основой для исследования случайного спектра симметрической булевой функции при различных конкретизациях вероятностной меры $\mathbf{P}$, и в качестве таковой здесь мы рассматриваем случай, когда мера $\mathbf{P}$ сосредоточена на подмножестве

$$
S_{n r}=\left\{\bar{f}_{n}:\left\|\bar{f}_{n}\right\|=r\right\}
$$

при некотором $r, 0<r<n$, и на этом подмножестве она равномерна:

$$
\mathbf{P}\left(\bar{f}_{n}\right)=\left(\begin{array}{c}
n+1 \\
r
\end{array}\right)^{-1}, \quad \bar{f}_{n} \in S_{n r} .
$$

В этом случае мы будем говорить о случайной симметрической булевой функции ранга $r$, а саму модель будем кратко называть моделью $S_{n r}$. Для этого случая формула (50) принимает вид

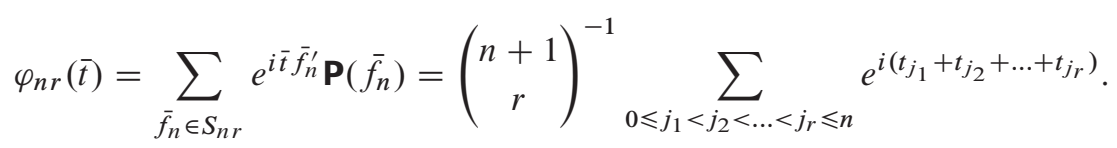

Здесь многомерная сумма может быть записана как коэффициент при $z^{r}$ произведения $\prod_{j=0}^{n}\left(1+z e^{i t_{j}}\right)$, поэтому окончательно получаем, что

$$
\varphi_{n r}(\bar{t})=\left(\begin{array}{c}
n+1 \\
r
\end{array}\right)^{-1} g\left[z^{r}\right] \prod_{j=0}^{n}\left(1+z e^{i t_{j}}\right)
$$

a (cм. (51))

$$
\Phi_{n r}(\bar{t})=\left(\begin{array}{c}
n+1 \\
r
\end{array}\right)^{-1}\left[z^{r}\right] \prod_{k=0}^{n}\left(1+z \exp \left\{i \sum_{m=0}^{n} t_{m} P_{k}(m ; n)\right\}\right)
$$

Представление (52) и является основой для дальнейшего анализа.

\section{2. Моменты спектра}

Общие формулы для первых и вторых моментов случайного спектра $\bar{w}_{n}=\left(w_{0}, \ldots, w_{n}\right)$ в рассматриваемой модели следуют из представления (48):

$$
\begin{aligned}
\mathbf{E} \bar{w}_{n} & =\mathbf{E} \bar{f}_{n} P_{n}, \\
\mathbf{D} \bar{w}_{n}=\left(\operatorname{cov}\left(w_{l}, w_{m}\right)\right)_{0}^{n} & =P_{n}^{\prime} \mathbf{D} \bar{f}_{n} P_{n} .
\end{aligned}
$$


Здесь для любого $j$

$$
\mathbf{E} f_{j}=\mathbf{P}\left(f_{j}=1\right)=\left(\begin{array}{c}
n \\
r-1
\end{array}\right) /\left(\begin{array}{c}
n+1 \\
r
\end{array}\right)=\frac{r}{n+1},
$$

и при $j \neq s$

$$
\mathbf{E} f_{j} f_{s}=\mathbf{P}\left(f_{j}=f_{s}=1\right)=\left(\begin{array}{c}
n-1 \\
r-2
\end{array}\right) /\left(\begin{array}{c}
n+1 \\
r
\end{array}\right)=\frac{r(r-1)}{n(n+1)} \text {. }
$$

Отсюда

$$
\begin{gathered}
\mathbf{D} f_{j}=\mathbf{E} f_{j}-\left(\mathbf{E} f_{j}\right)^{2}=\frac{r(n-r+1)}{(n+1)^{2}}, \\
\operatorname{cov}\left(f_{j}, f_{s}\right)=\mathbf{E} f_{j} f_{s}-\mathbf{E} f_{j} \mathbf{E} f_{s}=-\frac{r(n-r+1)}{n(n+1)^{2}}, \quad j \neq s .
\end{gathered}
$$

Обозначив

$$
a_{n r}=\frac{r(n-r+1)}{(n+1)^{2}}
$$

дисперсионную матрицу случайного вектора $\bar{f}_{n}$ можно, следовательно, записать в виде

$$
\begin{aligned}
\mathbf{D} \bar{f}_{n} & =a_{n r}\left(I_{n+1}-\frac{1}{n}\left(E_{n+1}-I_{n+1}\right)\right. \\
& =a_{n r}\left(\frac{n+1}{n} I_{n+1}-\frac{1}{n} E_{n+1}\right) \equiv a_{n r} B_{n},
\end{aligned}
$$

где, напомним, $I_{k}$ есть единичная матрица порядка $k$, а $E_{k}$ - матрица порядка $k$, все элементы которой равны 1.

Учитывая, что

$$
P_{k}(0 ; n)=\left(\begin{array}{l}
n \\
k
\end{array}\right)
$$

а при $m>0$

$$
\sum_{k} P_{k}(m ; n)=0
$$

находим средние значения спектра:

$$
\mu_{m}(n)=\mathbf{E} w_{m}=\frac{r}{n+1} \sum_{k=0}^{n} P_{k}(m ; n)=\frac{r}{n+1} \cdot \begin{cases}2^{n}, & m=0 \\ 0, & m>0\end{cases}
$$

Далее, из (53) и (54) находим

$$
\mathbf{D} \bar{w}_{n}=a_{n r} P_{n}^{\prime} B_{n} P_{n}=a_{n r}\left(\frac{n+1}{n} P_{n}^{\prime} P_{n}-\frac{1}{n} P_{n}^{\prime} E_{n+1} P_{n}\right) .
$$


Здесь

$$
P_{n}^{\prime} P_{n}=\left\|\sigma_{l m}(n)\right\|,
$$

где

$$
\sigma_{l m}(n)= \begin{cases}0, & \text { если } l+m \text { нечетно, } \\ (-1)^{m+(l+m) / 2} \frac{2^{2 n}}{\pi} B\left(\frac{l+m+1}{2}, n-\frac{l+m-1}{2}\right), & \text { если } l+m \text { четно, }\end{cases}
$$

и $B(a, b)$ - бета-функция:

$$
B(a, b)=\int_{0}^{1} t^{a-1}(1-t)^{b-1} d t, \quad a>0, \quad b>0 .
$$

Наконец, прямая проверка дает, что

$$
P_{n}^{\prime} E_{n+1} P_{n}=2^{2 n} C_{n+1},
$$

где матрица $C_{n+1}$ состоит из нулей, за исключением первого элемента, который равен 1. В итоге получаем следующее представление для дисперсионной матрицы спектра:

$$
\mathbf{D} \bar{w}_{n}=a_{n r}\left(\frac{n+1}{n}\left\|\sigma_{l m}(n)\right\|-\frac{2^{2 n}}{n} C_{n+1}\right) .
$$

Подведем итог в виде следующего утверждения.

Теорема 9. В модели $S_{n r}$ элементы спектра $\bar{w}_{n}=\left(w_{0}, w_{1}, \ldots, w_{n}\right)$ имеют нулевые средние значения, за исключением элемента $w_{0}$, см. (55), элементы с номерами разной четности некоррелированы, см. (56), а для дисперсий справедливы представления

$$
d_{m}^{2}(n)=\mathbf{D} w_{m}=a_{n r} \cdot \begin{cases}\frac{n+1}{n} \sigma_{0}^{2}(n)-\frac{2^{2 n}}{n}, & m=0, \\ \frac{n+1}{n} \sigma_{m}^{2}(n), & m>0,\end{cases}
$$

где

$$
\sigma_{m}^{2}(n)=\frac{2^{2 n}}{\pi} B\left(m+\frac{1}{2}, n-m+\frac{1}{2}\right),
$$

при этом $\sigma_{n-m}^{2}(n)=\sigma_{m}^{2}(n)$.

Подчеркнем, что зависимость дисперсий от параметра $r$ проявляется лишь через коэффициент

$$
a_{n r}=\frac{r(n-r+1)}{(n+1)^{2}}
$$

В дальнейшем мы будем проводить асимптотический анализ модели $S_{n r}$ в следующих условиях:

$$
n \rightarrow \infty, \quad r=r(n) \rightarrow \infty, \quad 0<\alpha=r / n<1 .
$$


Используя асимптотическую формулу Стирлинга для гамма-функции

$$
\Gamma(x+1) \sim x^{x} e^{-x} \sqrt{2 \pi x}, \quad x \rightarrow \infty,
$$

из (58)-(59) получаем, что в условиях (60) при фиксированном $m$

$$
d_{m}^{2}(n) \sim \frac{\alpha(1-\alpha)}{\pi} \Gamma\left(m+\frac{1}{2}\right) \frac{2^{2 n}}{n^{m+1 / 2}},
$$

и

$$
d_{m}^{2}(n) \sim \alpha(1-\alpha) \sqrt{\frac{2}{\pi n}} 2^{2 n} m^{m}(n-m)^{n-m} n^{-n},
$$

если $n, m, n-m \rightarrow \infty$.

Отметим также следующие свойства дисперсий, являющиеся прямым следствием известных свойств бета-функции (см. (59)):

(1) симметрия: $d_{m}^{2}(n)=d_{n-m}^{2}(n), m>0$,

(2) монотонность: $d_{m}^{2}(n)>d_{m+1}^{2}(n), 0 \leqslant m \leqslant n / 2-1$.

Далее, нетрудно убедиться в том, что с ростом $m$ дисперсии весьма быстро убывают, достигая своего минимального значения, асимптотически равного $\alpha(1-\alpha) \sqrt{2 /(\pi n)} 2^{n}$, при $m=[n / 2]$.

Рассмотрим, наконец, коэффициент корреляции

$$
\rho_{l m}(n)=\frac{\operatorname{cov}\left(w_{l}, w_{m}\right)}{\mathbf{D} w_{l} \mathbf{D} w_{m}} .
$$

Из (56)-(59) имеем, что при $0<l<m$ и четном $l+m$ (при нечетном $l+m$ коэффициент корреляции равен нулю)

$$
\begin{aligned}
\rho_{l m}(n) & =\frac{\sigma_{l m}(n)}{\sigma_{l}(n) \sigma_{m}(n)} \\
& =\frac{(-1)^{l+(l+m) / 2} B\left(\frac{l+m+1}{2}, n-\frac{l+m-1}{2}\right)}{\sqrt{B\left(l+\frac{1}{2}, n-l+\frac{1}{2}\right) B\left(m+\frac{1}{2}, n-m+\frac{1}{2}\right)}} .
\end{aligned}
$$

Применение формулы Стирлинга дает при $n \rightarrow \infty$ и фиксированном $m$

$$
\begin{aligned}
\lim \rho_{l m}(n) & =\rho_{l m} \\
& =(-1)^{l+(l+m) / 2} \frac{\Gamma\left(\frac{l+m+1}{2}\right)}{\sqrt{\Gamma\left(l+\frac{1}{2}\right) \Gamma\left(m+\frac{1}{2}\right)}}
\end{aligned}
$$

(этот результат справедлив и при $l=0$, так как (см. (58)) добавление $2^{2 n} / n$ на асимптотику дисперсии $d_{0}^{2}(n)$ (см. (61)) влияния не оказывает). Если здесь, в свою очередь, и $m \rightarrow \infty$ ( $l$ остается фиксированным), то правая часть в (62) стремится к нулю, имея порядок $O\left(m^{1 / 2} 2^{-m / 2}\right)$, то есть корреляция между спектральными коэффициентами $w_{l}$ и $w_{m}$ неограниченно уменьшается с увеличением расстояния $m-l$ между ними. 
Рассмотрим, например, корреляцию между крайними симметричными коэффициентами $w_{l}$ и $w_{n-l}, l \geqslant 0, n$ четно. В силу свойства симметрии дисперсии (при $l=0$ это свойство выполняется асимптотически при $n \rightarrow \infty$ )

$$
\rho_{l, n-l}(n)=(-1)^{l+n / 2} \frac{B\left(\frac{n+1}{2}, \frac{n+1}{2}\right)}{B\left(l+\frac{1}{2}, n-l+\frac{1}{2}\right)}=O\left(\frac{n^{l}}{2^{n}}\right),
$$

так что этот коэффициент корреляции весьма быстро стремится к нулю, когда $n \rightarrow \infty$ (этот результат понадобится нам в дальнейшем).

\section{3. Распределения спектральных коэффициентов}

Перейдем теперь к вопросу о распределениях спектральных характеристик в модели $S_{n r}$ и, прежде всего, найдем распределение отдельных компонент вектора $\bar{w}_{n}$. Для характеристической функции произвольного спектрального коэффициента $w_{m}, m \geqslant 0$, из общей характеристической функции (52) получаем представление

$$
\mathbf{E} e^{i t_{m} w_{m}}=\left.\Phi_{n r}(\bar{t})\right|_{\substack{t_{j=0}=0 \\
j \neq m}}=\left(\begin{array}{c}
n+1 \\
r
\end{array}\right)^{-1}\left[z^{r}\right] \prod_{k=0}^{n}\left(1+z \exp \left\{i t_{m} P_{k}(m ; n)\right\}\right) .
$$

Отсюда можно записать явное выражение характеристической функции в виде

$$
\mathbf{E} e^{i t w_{m}}=\left(\begin{array}{c}
n+1 \\
r
\end{array}\right)^{-1} \sum_{0 \leqslant k_{1}<\ldots<k_{r} \leqslant n} \exp \left\{i t\left(P_{k_{1}}(m ; n)+\ldots+P_{k_{r}}(m ; n)\right)\right\},
$$

из которого сразу следует, что случайная величина $w_{m}$ имеет равномерное распределение на множестве $\left\{P_{k_{1}}(m ; n)+P_{k_{2}}(m ; n)+\ldots+P_{k_{r}}(m ; n), 0 \leqslant k_{1}<k_{2}<\ldots<k_{r} \leqslant n\right\}$. В частности, первый спектральный коэффициент $w_{0}$ принимает с одинаковыми вероятностями $\left(\begin{array}{c}n+1 \\ r\end{array}\right)^{-1}$ все значения вида $\left(\begin{array}{c}n \\ k_{1}\end{array}\right)+\left(\begin{array}{c}n \\ k_{2}\end{array}\right)+\ldots+\left(\begin{array}{c}n \\ k_{r}\end{array}\right), 0 \leqslant k_{1}<k_{2}<\cdots<k_{r} \leqslant n$.

Аналогично можно выписать в явном виде и совместные (двумерные и т. д.) распределения спектральных коэффициентов, но они, очевидно, мало пригодны для практического использования. Более информативными в рассматриваемой модели являются соответствующие асимптотические распределения, к выводу которых мы и переходим. Итак, далее мы будем предполагать выполненными асимптотические условия (60).

Прежде всего, мы установим следующее ключевое свойство модели $S_{n r}$.

Лемма 1. В модели $S_{n r}$ распределение вектора $\bar{f}_{n}=\left(f_{0}, f_{1}, \ldots, f_{n}\right)$ совпадает с условнымм распределением независимых и одинаково распределенных бернуллиевских случайных величин $\xi_{0}, \xi_{1}, \ldots, \xi_{n}$ с произвольным параметром при условии, что их сумма равна $r$.

Доказательство. Действительно, распределение вектора $\bar{f}_{n}=\left(f_{0}, f_{1}, \ldots, f_{n}\right)$ сосредоточено на множестве двоичных векторов веса $r$ и оно равномерное. В свою очередь, если существует представление

$$
\mathbf{P}\left(\xi_{j}=\varepsilon\right)=\alpha^{\varepsilon}(1-\alpha)^{1-\varepsilon}, \quad \alpha \in(0,1),
$$


TO

$$
\begin{aligned}
\mathbf{P}\left(\xi_{j}=\varepsilon_{j}, j=0,1, \ldots, n \mid \sum_{j} \xi_{j}=r\right) & =\frac{\mathbf{P}\left(\xi_{j}=\varepsilon_{j}, j=0,1, \ldots, n, \sum_{j} \xi_{j}=r\right)}{\mathbf{P}\left(\sum_{j} \xi_{j}=r\right)} \\
& =\frac{\alpha^{r}(1-\alpha)^{n+1-r}}{\left(\begin{array}{c}
n+1 \\
r
\end{array}\right) \alpha^{r}(1-\alpha)^{n+1-r}}=\left(\begin{array}{c}
n+1 \\
r
\end{array}\right),
\end{aligned}
$$

что и доказывает лемму.

Таким образом, компоненты вектора $\bar{f}_{n}=\left(f_{0}, f_{1}, \ldots, f_{n}\right)$ являются в нашей модели условно независимыми случайными величинами, а спектральные коэффициенты (см. (45)) представляют собой линейные разделимые статистики от них (см. [7]), асимптотическая теория которых хорошо разработана. Применение центральной предельной теоремы для разделимых статистик, которая справедлива при условии, что дисперсия статистики неограниченно возрастает - эти условия у нас выполняются, позволяет сформулировать следующий основной результат.

Теорема 10. Пусть выполняются условия (60). Тогда распределение произвольного спектрального коэффициента $w_{m}, m \geqslant 0-$ фиксировано, асимптотически нормально с параметрами $\mu_{m}(n), c м .(55), u d_{m}^{2}(n), c м .(61)$.

Замечание 1. В силу свойства симметрии дисперсий $d_{m}^{2}(n)$ (для $m=0$ это свойство выполняется асимптотически), такое же утверждение справедливо и для симметричного коэффициента $w_{n-m}$.

Замечание 2. Свойство асимптотической нормальности при сформулированных условиях имеет место и для произвольных конечномерных распределений спектральных коэффициентов в рассматриваемой модели. Отметим при этом следующую особенность этой модели: нормировки корнем из дисперсий в соответствующей предельной нормальной теореме имеют разный порядок для разных спектральных коэффициентов. Именно, как следует из соотношения (61), при $n \rightarrow \infty$ и ограниченных значениях $m$ дисперсии $d_{m}^{2}(n)=\mathbf{D} w_{m}$ весьма быстро убывают с увеличением $m$ :

$$
\frac{d_{m+1}(n)}{d_{m}(n)}=O\left(n^{-1 / 2}\right) \text {. }
$$

Отсюда следует, что спектральные коэффициенты весьма сильно “разделены”. Например, обозначив

$$
w_{m}^{*}=\frac{w_{m}-\mu_{m}(n)}{d_{m}(n)}, \quad m=0,1, \ldots, n,
$$

получаем (далее аргумент $n$ для краткости опускаем):

$$
\mathbf{P}\left(\left|w_{m+1}-\mu_{m+1}\right|<\left|w_{m}-\mu_{m}\right|\right)=\mathbf{P}\left(\left|w_{m+1}^{*}\right|<\left|w_{m}^{*}\right| \frac{d_{m}}{d_{m+1}}\right) \rightarrow 1 .
$$

В частности, это означает, что максимальными среди спектральных коэффициентов являются крайние из них. 
Более детально эти важные для приложений вопросы мы обсудим ниже.

Будем под $w_{0}$ понимать далее центрированную величину $w_{0}-2^{n} r /(n+1)$, остальные спектральные коэффициенты в нашем случае центрированы в нуле, и рассмотрим следующую характеристику спектра:

$$
\Delta_{m}(n)=\frac{1}{d_{m}} \max _{m \leqslant k \leqslant n-m}\left|w_{k}\right|, \quad m \geqslant 0 .
$$

Как следует из теоремы 10 и замечания 2, в силу асимптотической некоррелированности, а следовательно, и асимптотической независимости крайних спектральных коэффициентов, при $n \rightarrow \infty$ и ограниченных значениях $m$ предельное распределение величины

$$
\delta_{m}(n)=\max \left(\left|w_{m}^{*}\right|,\left|w_{n-m}^{*}\right|\right)
$$

существует и имеет вид

$$
\left.\mathbf{P}\left(\delta_{m}(n)<x\right)\right)=\mathbf{P}\left(\left|w_{m}^{*}\right|<x,\left|w_{n-m}^{*}\right|<x\right) \rightarrow(2 \Phi(x)-1)^{2}, \quad x>0,
$$

где $\Phi(x)$ - функция стандартного нормального распределения.

Такое же предельное распределение в этих условиях имеет и определенная в (63) характеристика. Чтобы установить это, достаточно показать, что величина

$$
\Delta_{m}^{\prime}(n)=\frac{1}{d_{m}} \max _{m+1 \leqslant k \leqslant n-m-1}\left|w_{k}\right|
$$

стремится по вероятности к нулю. Используя неравенство Чебышева, получаем, что

$$
\begin{aligned}
\left.\mathbf{P}\left(\Delta_{m}^{\prime}(n)>\varepsilon\right)\right) & =\mathbf{P}\left(\bigcup_{k=m+1}^{n-m-1}\left\{\left|w_{k}\right|>\varepsilon d_{m}\right\}\right) \\
& \leqslant \sum_{k=m+1}^{n-m-1} \mathbf{P}\left(\left|w_{k}\right|>\varepsilon d_{m}\right) \leqslant \sum_{k=m+1}^{n-m-1} \frac{d_{k}^{2}}{\varepsilon^{2} d_{m}^{2}} .
\end{aligned}
$$

Из (61) и свойства монотонности дисперсий следует, что

$$
\frac{d_{k}^{2}}{d_{m}^{2}}= \begin{cases}O\left(n^{-1}\right) & \text { при } k=m+1 \text { и } k=n-m-1, \\ O\left(n^{-2}\right) & \text { при } m+2 \leqslant k \leqslant n-m-2 .\end{cases}
$$

Поэтому вся сумма в правой части (66) есть величина $O\left(n^{-1}\right)$, что и требовалось показать. Сформулируем итоговое утверждение.

Теорема 11. При $n \rightarrow \infty$ июбом фиксированном $m \geqslant 0$ в условиях (60) случайная величина (63) имеет предельную функцию распределения $(2 \Phi(x)-1)^{2}, x>0$.

Замечание 3. Аналогичными рассуждениями можно также установить, что если рассмотреть характеристику

$$
\Delta_{m, s}(n)=\frac{1}{d_{\min (m, s)}} \max _{m \leqslant k \leqslant n-s}\left|w_{k}\right|, \quad m \neq s,
$$

то при любых фиксированных $m$ и $s$ она имеет предельную функцию распределения $2 \Phi(x)-1, x>0$, такую же, как и случайная величина $\left|w_{\min (m, s)}^{*}\right|$. 
Эти результаты позволяют заключить, что с вероятностью, стремящейся к 1, максимальными среди всех (либо части) спектральных коэффициентов являются крайние из них. Если же говорить более конкретно, то структура спектра $\bar{w}_{n}=\left(w_{0}, w_{1}, \ldots, w_{n}\right)$ случайной симметрической булевой функции в модели $S_{n r}$ и при выполнении условий (60) устроена асимптотически следующим образом.

Максимальным коэффициентом является $w_{0}$ : он принимает значения в окрестности точки $\alpha 2^{n}$, а размер этой окрестности имеет порядок $O\left(2^{n} / n^{1 / 4}\right)$; все остальные коэффициенты принимают значения в окрестностях нуля, причем размеры соответствующих окрестностей резко различаются: для $w_{n}$ разброс имеет порядок $O\left(2^{n} / n^{1 / 4}\right)$, для $w_{1}$ и $w_{n-1}$ - порядок $O\left(2^{n} / n^{3 / 4}\right)$, для $w_{2}$ и $w_{n-2}-$ порядок $O\left(2^{n} / n^{5 / 4}\right)$, и т. д.

\section{Список литературы}

1. Сачков В. Н., Введение в комбинаторные методы дискретной математики. МЦНМО, Москва, 2004.

2. Логачев О. А., Сальников А. Л., Ященко В. В., Булевы функции в теории кодирования и криптологии. МЦНМО, Москва, 2004.

3. Cusick T. W., Stănică P., Cryptographic Boolean functions and applications. Elsevier, Amsterdam, 2009.

4. Ивченко Г. И., Медведев Ю. И., Спектр случайной булевой функции и его производящая функция. Математические вопросы криптографии (2011) 2, №2, 41-54.

5. Ивченко Г. И., Медведев Ю. И., Стохастические булевы функции и их спектры. Математические вопросы криптографии (2012) 3, №3, 21-34.

6. Сегё Г., Ортогональные многочлены. Физматлит, Москва, 1962.

7. Ивченко Г. И., Иванов В. А., Медведев Ю. И., Дискретные задачи в теории вероятностей. Итоги науки и техники. Сер. Теория вероятн., матем. статист., теор. киберн. (1984) 22, 3-60.

8. Canteaut A., Videau M., Symmetric Boolean functions. IEEE Trans. Inform. Theory (2005) 51, 2791-2811.

9. Peng J., Wu Q., Kan H., On symmetric Boolean functions with high algebraic immunity on even number of variables. IEEE Trans. Inform. Theory (2011) 57, 7205-7220.

Статья поступила 06.02.2013. 